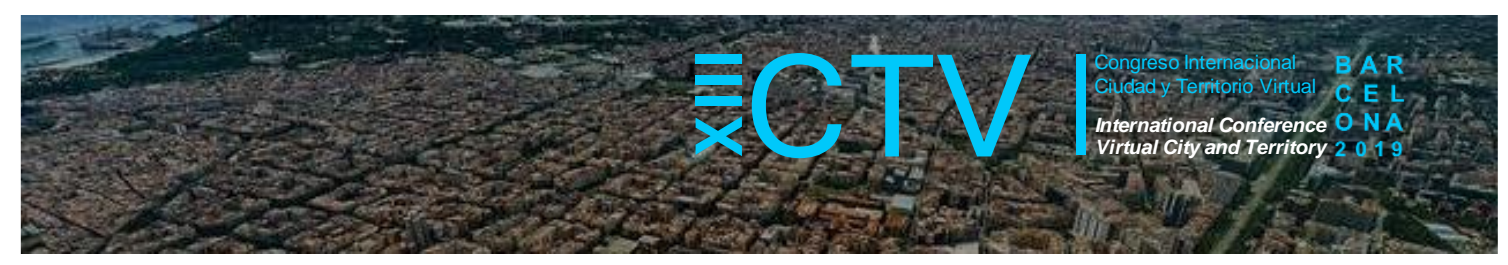

\title{
LA AGENDA PARA UN FUTURO DIGITAL Y ECOLÓGICO
}

\author{
Charlier, Marike ${ }^{1 *}$; Francou, Renaud ${ }^{2}$
}

Remisión inicial: 2019-07-04; Remisión definitiva: 2019-10-27; Publicación: 2019-21-12

Citación: Charlier, M. et al. (2019). La agenda para un futuro digital y ecológico. En XIII CTV 2019 Proceedings: XIII International Conference on Virtual City and Territory: "Challenges and paradigms of the contemporary city”: UPC, Barcelona, October 2-4, 2019. Barcelona: CPSV, 2019, p. 8749. EISSN 2604-6512. DOI http://dx.doi.org/10.5821/ctv.8749

\section{Resumen}

La ecología suele criticar los impactos de la digitalización, símbolo de aceleración global y mundialización de los flujos, mientras el motor de los actores de la digitalización son las oportunidades de creación de valor económica antes que cualquier consideración de sus riesgos o impactos.

En base a estas consideraciones, la convergencia entre los dos sectores parece muy difícil. Sin embargo, la Fundación Internet Nueva Generación (FING), un think tank francés que define su misión como imaginar concretamente una digitalización con futuro y centrada en las capacidades humanas, observó lo siguiente:

"La transición ecológica es el horizonte de nuestras sociedades y la transición digital, la gran fuerza transformadora de nuestra época. La primera conoce su destino, pero le cuesta dibujar su camino; la segunda es nuestro día a día; una fuerza permanente de cambio pero que no persigue ningún objetivo colectivo específico. Una tiene el objetivo, la otra el camino: jestas dos transiciones se necesitan la una a la otra!" Con esta idea nació, en 2015, el proyecto de la Agenda para un futuro digital y ecológico*. Se publicó en marzo 2019, después de 3 años de trabajos colaborativos, en asociación con la ADEME o el IDDRI entre otros y se dirige a investigadores, empresas, organismos, actores públicos, medias, etc., para que difundan $\mathrm{y} / \mathrm{o}$ se apropien sus recomendaciones.

Esta Agenda busca la colaboración entre lo digital y lo ecológico apoyándose en la convicción de que la transformación digital no tiene sentido, ni futuro, si no considera su propia huella ecológica y no se moviliza para actuar en contra del cambio climático y las demás consecuencias de la actividad humana. Para lograr esta convergencia, marca nuevos caminos o caminos a potenciar que se fomentan en 5 principios (que la agenda detalla en 20 retos):

1. La tecnología no aporta soluciones, pero las soluciones pueden producir tecnologías.

Para contribuir a la transición ecológica, la digitalización debe participar en modelos alternativos de desarrollo, otras formas de producción, intercambio y consumo.

2. La fuerza transformadora de la digitalización no está en los cálculos sino en la acción colectiva.

Los datos son imprescindibles para muchas acciones medioambientales, pero sólo una gestión abierta, de uso compartido y generadora de interacción social, permitirá movilizar los colectivos ciudadanos y tener un impacto transformador.

3. La digitalización colaborativa y la ecología democrática son indisociables, aunque todavía no son plenamente conscientes de ello.

Los modelos digitales han demostrado su fuerza transformadora pero su contribución a la ecología no es nada evidente. El acercamiento entre los actores de la digitalización colaborativa, los de la innovación pública y democrática y los ecológicos permitirá, aprendiendo unos de los otros, generar una cultura común de los retos digitales y medioambientales.

4. La innovación sólo podrá tener un papel positivo en la transición ecológica si se concentra tanto en su impacto como en su modelo económico.

El sistema de gestión de la innovación, las instituciones y dispositivos financieros que la permiten, deben controlar que la innovación mida sus impactos ambientales y sociales y asegurarse que realmente consiga sus objetivos en la materia.

5. La digitalización y su industria deben enseñar el camino

\footnotetext{
${ }^{1}$ Ingeniera industrial con Master en Planificación Urbana y Sostenibilidad, Universidad Politécnica de Cataluña, ${ }^{2}$ Fondation Internet Nouvelle Génération (FING). * Correo de contacto: marike.charlier@gmail.com
} 


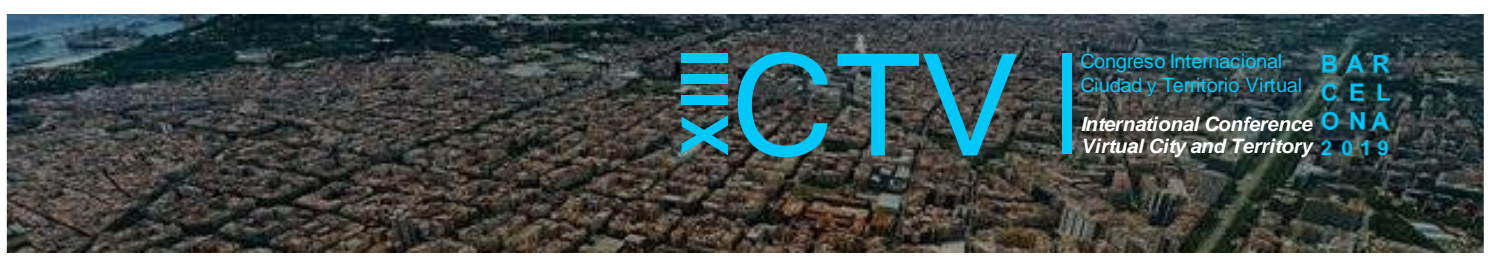

La industria digital debe ser ecológica por diseño y liderar la economía circular, proponiendo productos ecoresponsables, reparables o reciclables, con una vida más larga y menos consumidores de recursos.

Los retos de la Agenda para un futuro digital y ecológico marcan los pasos para conseguir una digitalización innovadora a la vez que capacitadora, frugal, democrática, equitativa y factor de confianza. No cabe duda de que el potencial ecológico de esta digitalización es considerable y si consideramos que la problemática es mundial, interesa que el mensaje de la Agenda se propague y se aplique fuera de los límites del hexágono.

\section{Abstract}

Ecology often criticizes the impacts of digitization considering it as a symbol of global acceleration and globalization of flows. Moreover, there is little doubt that digitization actors are rather moved by opportunities related to economic value creation than any consideration about their risks or impacts.

Based on these considerations, the convergence between the two sectors seems very difficult. However, the Internet New Generation Foundation (FING), a French think tank that defines its mission as concretely imagining a digitization with a future and focused on human capabilities, observed the following:

"The ecological transition is the horizon of our societies and the digital transition, the great transforming force of our time. The first knows her destination, but it is hard for her to draw her way; the second is our day to day; a permanent force of change but that does not pursue any specific collective objective. One has the objective, the other the way: these two transitions need each other!" With this idea, the project of an Agenda for a digital and ecological future* was born in 2015. It was published in March 2019, after 3 years of collaborative work, in association with ADEME or IDDRI among others. It is aimed at researchers, companies, organizations, public actors, media, etc., to disseminate their recommendations.

This Agenda seeks collaboration between digitization and ecology based on the conviction that digital transformation has no meaning, nor future, if it does not consider its own ecological footprint and does not mobilize to act against climate change and the other consequences of human activity. To achieve this convergence, it marks new paths or existing paths to be reviewed or promoted that are classified in 5 principles (which the agenda details in 20 challenges):

1. Technology does not provide solutions, but solutions can produce technologies.

To contribute to the ecological transition, digitization must participate in alternative models of development, other forms of production, exchange and consumption.

2. The transformative force of digitization will not be a result of calculations but of collective action.

Data are essential for many environmental actions, but only their open management and shared use that generate social interaction, will allow the mobilization of citizen groups and a transformative impact.

3. Collaborative digitization and democratic ecology are inseparable, although they are not yet fully aware of it. Digital models have demonstrated their transformative force but their contribution to ecology is not obvious at all. The rapprochement between the actors of collaborative digitization, those of public and democratic innovation and ecological ones will generate a common culture of digital and environmental challenges thanks to shared learning.

4. Innovation can only play a positive role in the ecological transition if it focuses on both its impact and its economic model.

The innovation management system together with the institutions and financial devices that allow it, must control that innovation measures its environmental and social impacts and ensure that it really achieves its objectives in this matter.

5. Digitization and its industry must teach the way.

The digital industry must be green by design and lead the circular economy, proposing eco-responsible, repairable or recyclable products, with a longer life and lower resource consumption.

The challenges of the Agenda for a digital and ecological future mark the steps to achieve an innovative as well as frugal, democratic and trust builder digitization. There is no doubt that the ecological aptitude of this digitization is considerable. So, considering that the problem is worldwide, we consider the message of the Agenda has to be propagated and applied outside the limits of the hexagon.

Palabras Clave: Ecología; Digitalización; Open Source; Tech for Good

Key words: Ecology; Digitization; Open source; Tech for Good 


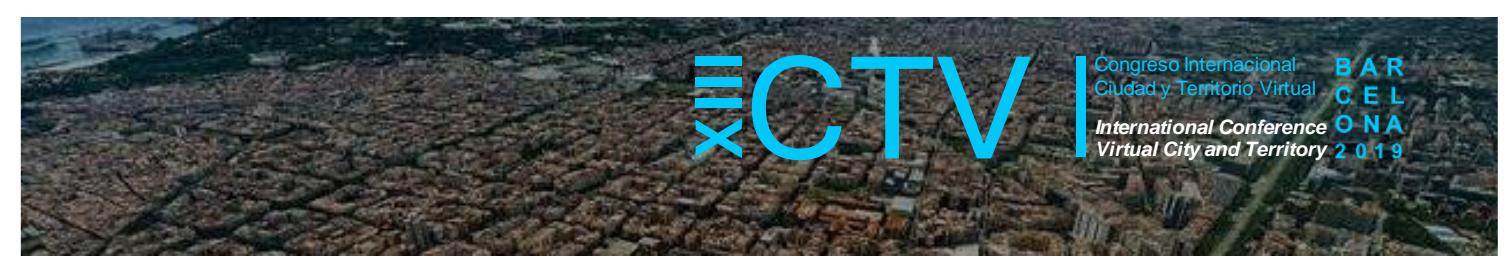

\section{Introducción}

El encuentro entre la digitalización y la ecología es, a priori, difícil. La ecología se basa, en parte, en una crítica de la tecnología y del crecimiento y no se fía de las promesas de innovación tecnológica: recalca los impactos ecológicos de la digitalización, a la vez que la considera, no sin razón, como herramienta y símbolo de la globalización y de la aceleración de los ritmos (de vida, de innovación, de obsolescencia...) y de los flujos (de materia, de energía, de residuos, ...).

Por su parte, los actores digitales suelen percibirse a sí mismos como inmateriales y eficientes, lo que los lleva a considerarse ecológicos por esencia. Guiados por una dinámica de innovación permanente, ven primero las oportunidades y, más tarde, los riesgos.

En 2015, la FING (Fundación del Internet Nueva Generación) lanzaba este reto: "La transición ecológica es el horizonte de nuestras sociedades y la transformación digital, la gran fuerza transformadora de nuestra época. La primera conoce su destino, pero le cuesta dibujar su camino; la segunda es nuestro día a día; una fuerza permanente de cambio pero que no persigue ningún objetivo colectivo específico. Una tiene el objetivo, la otra el camino: jestas dos transiciones se necesitan la una a la otra! Sin embargo, los actores actúan demasiado frecuentemente en ámbitos separados, sin percatarse de la potencia transformadora que tendría su convergencia."

De esta llamada, nació el proyecto colectivo Transitions², llevado por la FING con la ADEME, el IDDRI, INRIA, GreenIT.fr, el Consejo Nacional Digital francés, Explor'ables y otros, con el objetivo de "poner lo digital al servicio de la transición ecológica". Los propósitos de Transitions ${ }^{2}$ son explorar nuevas vías que permitan la convergencia entre digital y ecología - sin ocultar sus riesgos; dar sentido a la transición digital al confrontarla con los retos ecológicos; y descompartimentar las comunidades de actores provenientes respectivamente de la ecología y de la digitalización e iniciar acciones concretas que los acerquen.

Un resultado de los 3 primeros años de trabajos colaborativos de la iniciativa Transitions2 es la presente agenda para un futuro digital y ecológico que invita a los investigadores, innovadores, organizaciones pequeñas y grandes, actores públicos, militantes y medios de comunicación a implicarse en 3 direcciones:

1 Cambiar sus agendas respectivas: repasar sus prioridades y su manera de actuar gracias a intercambios con las comunidades del otro bando,

2 Dar una fuerza renovada a sus acciones gracias a la convergencia resultante,

3 Iniciar realizaciones comunes; eventos, proyectos, producciones que no existirían sin esta convergencia.

\section{Cinco principios para iniciar su propia agenda numérica y digital}

Aunque todavía hay muchas preguntas por resolver, ya se puede empezar a actuar para hacer converger las dos transiciones: la digital y la ecológica.

Para empezar, la digitalización debe concebir una agenda de innovación que tome en cuenta la finitud del mundo y la intrincación de las cuestiones ecológicas, tecnológicas, sociales y económicas. Para ganar credibilidad y potenciarse, debe primero reinventar su propia 


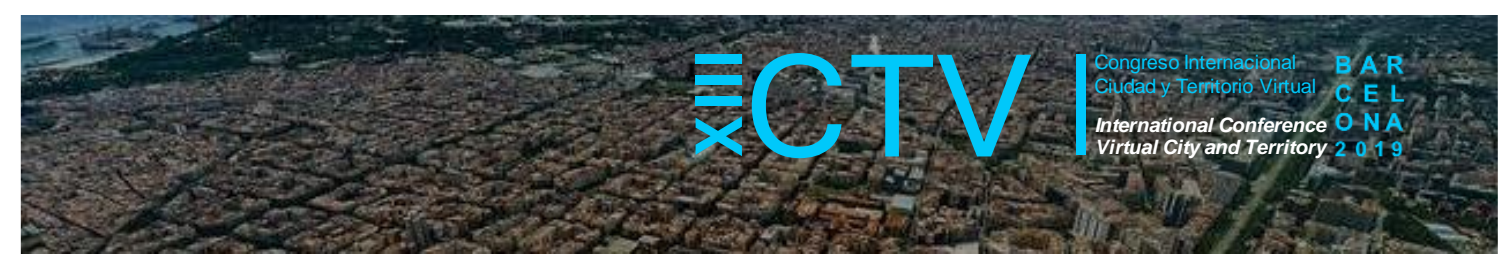

concepción, considerar seriamente su propia huella, "circularizar" su industria. Antes de modificar sus relaciones con el conjunto de actividades humanas que transforma, debe definir si su propósito es servir el modelo productivista y extractivista o permitir la emergencia de un mundo más sensible a los efectos de las acciones de unos sobre las posibilidades de existencia de otros. Por su parte, los actores de la transición ecológica deben apropiarse la fuerza de la transformación digital. Para ello, deben considerar lo digital como un poder de unión, una herramienta de capacitación tanto de los individuos como de los colectivos, una fuente de nuevos modelos en vez de un instrumento de cálculo reservado a unos pocos expertos.

Prácticamente, ¿cómo avanzar? ¿En qué dirección orientar esta agenda? Y, sobre todo, ¿cómo los investigadores, los innovadores, las empresas, los actores públicos, los medios de comunicación, ... pueden apropiarse de este trabajo?

Cualquiera sea el reto ecológico (del ámbito energético, agrario, de la movilidad u otro), que lo inicie un actor público, un organismo de investigación o un dispositivo de innovación, que se trate un tema emergente o más maduro, una agenda de la transformación digital al servicio de la transición ecológica debe apoyarse en 5 pilares:

\subsection{La tecnología no aporta soluciones, pero las soluciones pueden producir tecnologías}

Es cómodo considerar la cuestión ecológica como un conjunto de retos, problemas u objetivos que se podrían analizar uno por uno para proponer soluciones: es un sueño de ingeniero, fuente de muchas iniciativas bien intencionadas que recurren a las tecnologías (entre otras, digitales) para dar respuesta a los grandes retos de la humanidad. La digitalización suele ser la herramienta de medida y cálculo de estas acciones.

Por una parte, es una manera especialmente reducida de considerar la digitalización y, por otra parte, la cuestión ecológica es sistémica, los desafíos están inextricablemente enlazados y no se pueden tratar por separado. Si la digitalización debe ayudar a responder a la crisis ecológica, debe ser a través de otros modelos de desarrollo y de formas alternativas de producción, de consumo o de intercambio.

Dicho de otra manera, tal como lo subraya otro llamamiento de la FING - "RESET: Reinventar lo digital" - iniciado a principios de 2019, la digitalización tiene también que considerar los retos del mundo futuro y sólo podrá hacerlo transformándose en profundidad, en su propia lógica. Es en esta perspectiva que tienen que considerarse los aportes de las N-Tech (Tech for good, civic tech, food tech, low tech y otras) a los retos ecológicos.

Enfocar la digitalización al servicio del medio ambiente es, por lo tanto, menos una cuestión tecnológica que de actores, métodos organizativos, nuevos imaginarios.

Ilustraciones de este pilar:

- Reto $n^{\circ} 6$ : Los imaginarios como caminos de transición.

\footnotetext{
${ }^{2}$ Los retos se detallan en las categorías de agendas (capítulos II a IV)
} 


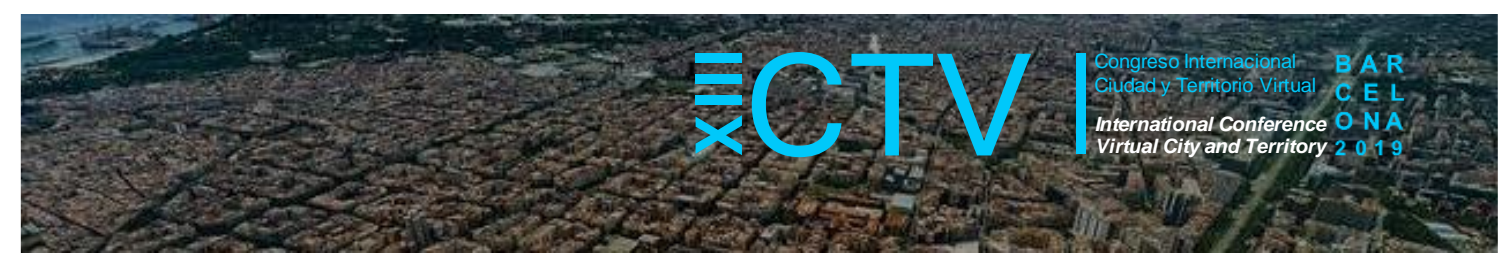

- Reto n`7: Construir una hoja de ruta de la ciudad sostenible e inteligente.

- Reto $n^{\circ} 8$ : Una "Industria del futuro" que toma en serio el medio ambiente.

- Reto $n^{\circ} 12:$ Nuevas fuentes de inspiración para la economía circular.

\subsection{La fuerza transformadora de la digitalización no está en el cálculo sino en la acción colectiva}

Los dispositivos ciudadanos de medida de calidad del aire (gracias a captores de bajo coste) sólo pueden cambiar los comportamientos si se desarrollan a escala colectiva: habitantes de un mismo edificio, barrio, compañeros de trabajo, o padres, por ejemplo. Los datos son una fuente importante de creación de conocimiento, pero, además, su uso dentro de las organizaciones ayuda a descompartimentar y romper las casillas. Las movilidades sostenibles son primero un tema de gobernanza, de orquestación de una multitud de iniciativas innovadoras y nuevas formas de colaboración entre actores privados y públicos.

La mayor fuerza de la digitalización al servicio de la transición ecológica no se encuentra en los cálculos, sino en el compartir, en la colaboración y en los vínculos sociales que generan. Los motores más eficaces de transformación de la digitalización están en la actuación colectiva.

Queda claro que una cultura común de los desafíos digitales y medioambientales es, más que nunca, necesaria. La digitalización es fuente de renovación de los imaginarios, sabe organizar la colaboración y el cambio de escala; la ecología, por su parte, sabe dotar de sentido la innovación, considera los efectos rebote, piensa en sistemas. Esta dimensión cultural - aprender unos de los otros y producir nuevas síntesis - constituye un preámbulo indispensable a cualquier iniciativa que quiera sacar partido en los dos ámbitos.

llustraciones de este pilar²:

- Reto n`3: La digitalización para un planteamiento colectivo de la movilidad sostenible.

- Reto $n^{\circ} 4$ : Poner los datos al servicio de los impactos medioambientales.

- Reto $n^{\circ} 5$ : Una medición colectiva de la calidad del aire.

- Reto $n^{\circ}$ 9: La digitalización al servicio de las políticas ambientales locales.

- Reto n`10: Estrategias para lugares compartidos.

- Reto n`18: La digitalización al servicio de la vivienda sostenible.

\subsection{La digitalización colaborativa y la ecología democrática tienen que ir de la mano, pero todavía no hay suficiente conciencia de ello}

Los modelos nacidos con la digitalización (Open, ágiles, compartidos, colaborativos, distribuidos, etc.) han demostrado su capacidad transformadora en muchos sectores, pero su aporte en la problemática ecológica no son nada evidentes. De momento, siguen siendo modelos: si no se les insufla un real objetivo ecológico, no darán resultados. Las promesas decepcionadas de la economía participativa nos lo recuerdan. Se puede, como Facebook, abrir su búsqueda en inteligencia artificial para acelerar el desarrollo de sistemas publicitarios cada vez más potentes; o, como Local Motors, concebir de manera colaborativa y simultanea unos buses eléctricos autónomos y unos $4 \times 4$ para los aficionados a los coches grandes. 


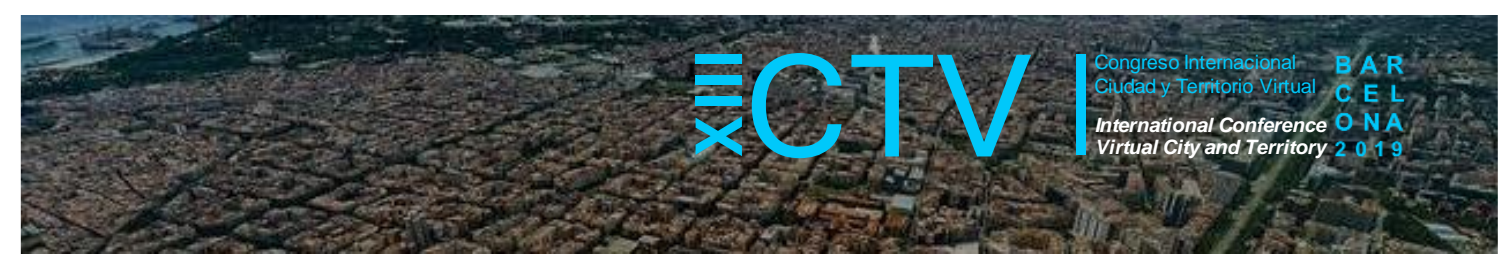

Obviamente todos los "civic techs" del mundo no bastarán para dar lugar a una democracia ecológica si los ciudadanos y las instituciones políticas (formales o informales) no comparten el desafío. Sin embargo, el mundo digital es, desde hace décadas, un lugar de experimentación social de formas colaborativas de toma de decisión y de acciones capaces de llevar la acción individual o local a gran escala (desde la contribución a Open Solar Map hasta las redes de Repair Cafés). Es uno de los espacios donde han florecido el pensamiento y la práctica de los comunes. Es un terreno real para la experimentación democrática. Un acercamiento estratégico entre los actores de la ecología, los de la innovación pública y democrática (digital o no) y los de la transformación digital colaborativa abriría nuevas perspectivas para la emergencia de une ecológica no técnica, que sea a la vez cotidiana y política.

Ilustraciones de este pilar:

- Reto n`11: Les "modelos abiertos" al servicio de la transición ecológica.

- Reto n०13: Conectar lo digital y el low tech.

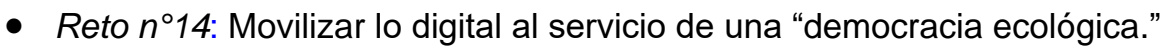

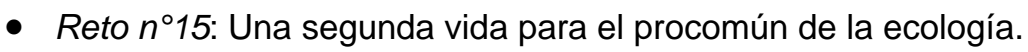

- Reto $n^{\circ}$ 17: Los aportes de la digitalización a la agricultura, la agro-ecología y la permacultura.

- Reto $n^{\circ}$ 19: Hacia el internet de la energía.

\subsection{La innovación sólo podrá jugar un papel positivo en la transición ecológica si considera tanto su impacto como su modelo económico}

Para conseguir la transición ecológica, necesitamos innovación, pero no una innovación cualquiera sino unos proyectos que busquen tener unos impactos medioambientales ambiciosos, explícitos y creíbles, que cuantifiquen su impacto en otros sectores y actores, y sobre todo que se doten de herramientas para averiguar que sus objetivos se cumplen ya que demasiadas pocas iniciativas las tienen a día de hoy.

Por otra parte, la innovación sólo tendrá un impacto positivo si el sistema de innovación - el conjunto de métodos, instituciones y dispositivos financieros que posibilitan, dan legitimidad y seleccionan las propuestas innovadoras - evoluciona para dar tanta importancia a los impactos (ecológicos y sociales, positivos y negativos) de los proyectos como a su creación de valor económico. Para conectar mejor el modelo de negocio de una innovación y su modelo de impacto, hay que apoyar y acompañar los innovadores que lo intentarán, sólo bajo esta condición se podrá conseguir.

Ilustración de este pilar²:

- Reto n²: Orientar la innovación hacia la búsqueda de un mayor impacto medioambiental.

\subsection{La digitalización y su industria deben enseñar el camino}

Las IT for Green no deben ser una excusa para abandonar las Green IT". Es decir que el sector digital debe reconsiderar su propia huella ecológica (que es masiva) antes que pretender dar soluciones para modelos, colectivos, ... La industria digital y sus usuarios deberían ser las cabezas visibles de la economía circular y proponer productos eco-responsables, modulares, 


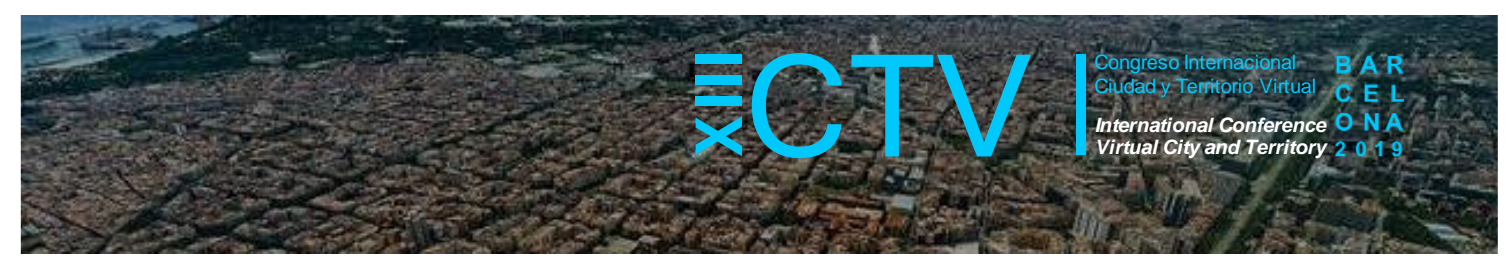

reparables, reciclables y sobre todo con una vida más larga, unos servicios pensados con una mente ahorradora, en recursos informáticos y de red, pero también en tiempo y atención.

Una agenda de lo digital al servicio del medio ambiente debe cuidar que la digitalización sea ecológica por diseño. La concepción digital debe revisar sus bases conceptivas: aclarar el proceso de toma de decisión y no oscurecerlo, simplificar la atención y no instrumentalizarla, repartir el poder de actuar y no prescribirlo, ...

La digitalización necesita un RESET: ¡revisar su contribución a la transición ecológica es la mejor manera de iniciar el cambio!

Ilustraciones de este pilar²:

- Reto n`1: Una digitalización ecológica por diseño.

- Reto $n^{\circ} 16$ : Una cultura común de lo digital y la ecología al servicio de los ODS (Objetivos de Desarrollo Sostenible).

- Reto n²0: Contra el colapso.

\section{Cinco agendas a iniciar}

\subsection{Reto $n^{\circ}$ 1: Una digitalización ecológica por diseño}

Lo digital tiene su propia huella ecológica que no se limita a su consumo energético. A lo largo de su ciclo de vida, las infraestructuras, los terminales y los servicios digitales consumen muchos recursos, y esta huella se incrementa de manera exponencial.

Para superar el reto de una digitalización ecológica por diseño, hace falta optimizar lo existente (limitar la huella ecológica de los servicios y productos que ya están en el mercado) pero también pensar en nuevos modelos de concepción, de desarrollo y de uso.

Para conseguirlo, las comunidades involucradas en el movimiento "GreenIT" tendrán que estructurarse para fortalecer su acción, actuar a la vez en dirección de los actores de la digitalización (infraestructuras, industriales, desarrolladores, servicios, ...) y de los usuarios sobre todo las empresas.

\subsection{Reto $n^{\circ} 2$ : Orientar la innovación hacia la consecución de un impacto medioambiental lo más reducido posible.}

Los sistemas de innovación deben permitir la emergencia y el crecimiento de proyectos que den tanta importancia al impacto ecológico y social como a la creación de valor económico. A pesar de las buenas intenciones, hoy no es el caso.

Para conseguirlo, se debe crear un repositorio para el análisis de proyectos con impacto, crear dispositivos específicos para su fomento, iniciar un análisis ecológico del conjunto de las políticas de ayuda a la innovación, para que, finalmente los métodos contables de las empresas evolucionen de manera a incluir el impacto de su actividad en los capitales no sólo financieros sino también naturales y humanos. 


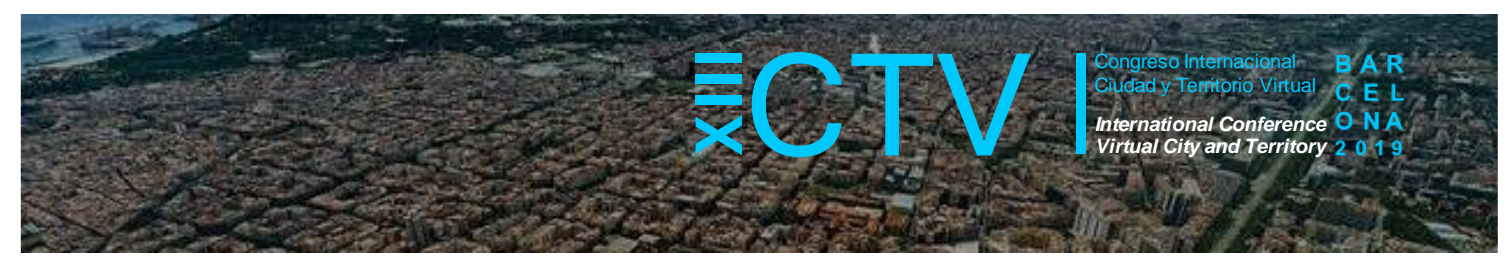

En Francia, la comunidad Transitions ${ }^{2}$ ha desarrollado un primer repositorio de la "Innovación Factor 4" que está en prueba en el periodo 2018-2019. Otras herramientas similares nacen o ya existen en el mundo, por lo que habrá que explorar vías de acercamiento para llegar a un "lenguaje común" a todos los innovadores y los que los acompañan.

\subsection{Reto $n^{\circ} 3$ : La digitalización para un planteamiento colectivo de la movilidad sostenible.}

La digitalización ha dado paso ya a muchas iniciativas relacionadas con la movilidad sostenible: optimizar los trayectos, incrementar la tasa de ocupación de los vehículos, diversificar las formas de movilidad, favorecer la movilidad colectiva y activa...

Sin embargo, las innovaciones en marcha no van todas en la misma dirección: enriquecen las ofertas existentes de movilidad, pero, a la vez, complican su gestión y gobernanza.

Para avanzar, los actores públicos podrían mejorar la comunicación acerca de sus intenciones medioambientales, trabajar a formas colectivas de innovación e inventar nuevas formas de gobernanza.

Mejoraría la innovación con una puesta en común de los medios y recursos para poder proponer respuestas conjuntas, más fáciles de gestionar para los actores públicos, los usuarios y los actores de la movilidad.

\subsection{Reto $n^{\circ}$ 4: Poner los datos al servicio de los impactos medioambientales.}

Los datos, como soportes significativos del conocimiento, de la toma de decisión y de la evaluación, pero también (bajo ciertas condiciones) del debate y de la colaboración, pueden jugar un papel decisivo al servicio de la acción medioambiental y ecológica. Pueden contribuir tanto a la toma de conciencia como a la exploración de soluciones.

Muchos proyectos públicos, privados y asociativos tienen este objetivo medioambiental, sin embargo, muchos conocen dificultades para durar en el tiempo, cambiar de escala y generar un impacto significativo. Parte de estas dificultades se deben a los juegos de poderes y de propiedad alrededor de los datos y la falta de cultura del dato compartido.

Las prioridades para la acción deben portar sobre el acceso a los datos, su coproducción y su reparto, sobre la cooperación entre los actores y finalmente sobre el desarrollo a gran escala del "data literacy" (la alfabetización de los datos) accesible a los no-especialistas.

\subsection{Reto $n^{\circ} 5$ : Una medición colectiva de la calidad del aire}

La era digital ha favorecido la emergencia de nuevas herramientas y maneras de medir la calidad del aire: iniciativas más o menos organizadas de "captores ciudadanos", dispositivos de medida, cartografía o data visualización...

Estas iniciativas de medición son, de momento, paralelas a los dispositivos de medición o producción de datos "históricos" y poco sirven para su verificación cuando podrían beneficiarse 


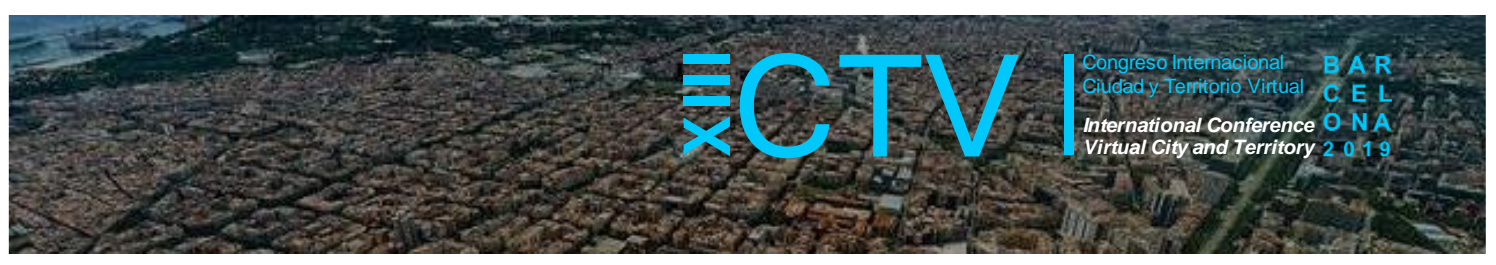

mutuamente con la comprensión aguda de las cuestiones complejas de la calidad del aire por un lado y la multitud y la implicación ciudadana por el otro.

El desafío consiste en gestionar la diversidad de las necesidades y, a la vez, dar sentido a un conjunto mucho más heterogéneo de datos.

Una agenda de la digitalización al servicio de la medición de la calidad del aire podría, por lo tanto, apoyarse sobre una base destinada a crear una cultura común entre los problemas de calidad del aire y los aspectos más técnicos relacionados con los captores y la medición en sí.

\section{Diez agendas a (re-)construir}

\subsection{Reto $n^{\circ} 6$ : Los imaginarios como caminos de transición}

La perspectiva tecnológica es la de la superación de los límites: se trata o de sobrepasarlos o de abolirlos. Al contrario, la ecología nos invita a integrar los límites: contener, reducir, conectar los sistemas en "bucles".

Muchos ejercicios de prospectiva y de ficción trabajan el enlace entre estas dos perspectivas, pero pocas se han comprometido con el objetivo explícito de actuar más allá de las soluciones tecnicistas.

El desafío consiste en considerar los imaginarios como capaces de abrir nuevas vías cuya exploración puede ser facilitada por la digitalización.

\subsection{Reto $n^{\circ}$ 7: Construir una hoja de ruta de la ciudad sostenible e inteligente}

Al convertir los sistemas urbanos en más eficientes, los proyectos de ciudad inteligente prometen, con demasiada facilidad, convertir las ciudades en más "verdes".

Sin embargo, los raíces y los actores fundadores respectivos de la Smart City (basada en la economía y los servicios) y la ciudad sostenible (que privilegia las dimensiones sociales y medioambientales) no son los mismos y tienen poca probabilidad de converger naturalmente.

El desafío consiste en acercar de manera voluntaria los proyectos de ciudad inteligente y de ciudad sostenible para construir una hoja de ruta común: métodos de concepción, indicadores, programas de apoyo, observatorios, ...

\subsection{Reto $n^{\circ}$ 8: Una "Industria del futuro" que se toma en serio el medioambiente}

Las proyecciones de la fábrica del futuro integran una digitalización muy amplia y técnica (robótica, inteligencia artificial, big data, ...) pero no suelen considerar la dimensión medioambiental como un reto primordial.

El desafío consiste en construir visiones comunes: actualmente no existe un relato común, cada actor cuenta su perspectiva a su manera y por separado. 


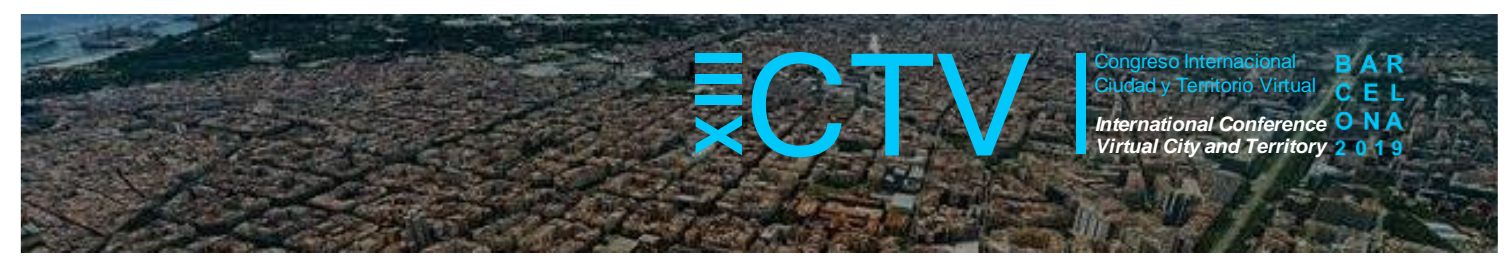

Para avanzar, hace falta construir una cultura común que integre todos los componentes: la cultura ingeniera, la cultura digital y la cultura medioambiental. Es la única manera para que la industria del futuro pueda sacar partido simultáneamente de la modularidad y agilidad de la innovación digital y a la vez pensarse como un elemento esencial de la transición ecológica.

\subsection{Reto $n^{\circ}$ 9: La digitalización al servicio de las políticas ambientales locales}

Que se trate de energía, de movilidad, de circuitos alimenticios cortos, de fabricación y reparación, de datos abiertos... los dispositivos digitales pueden resultar útiles para servir y reforzar las dinámicas locales, ayudarlas a conectar entre sí, facilitar la participación y la eficiencia.

Sin embargo, hoy todavía no es el caso, o lo es demasiado poco: las agendas 21, los planes de movilidad o de gestión de los residuos, etc., no se apoyan en las culturas digitales a pesar de que muchas innovaciones territoriales podrían ser utilizadas como plataforma.

Una agenda de lo digital al servicio de la actuación local debería intentar identificar las herramientas digitales que pueden suministrar a las iniciativas existentes el medio para conectar entre sí y facilitar la participación. Podrían gestionarse las innovaciones territoriales, los diseñadores de las políticas públicas podrían formarse en lo digital, podrían reforzarse la gestión de la movilidad, la autonomía energética, el buen funcionamiento de los circuitos cortos, etcetera.

\subsection{Reto $n^{\circ} 10$ : Estrategias para lugares compartidos}

La digitalización amenaza la existencia de algunos lugares que podrían quedarse obsoletos por la desmaterialización de unos cuantos servicios, pero a la vez genera dinámicas de reparto en lugares existentes y crea lugares colectivos basados en el compartir.

Estas dinámicas de reparto pueden coincidir con objetivos ecológicos: lucha contra el urban sprawl, disminución de los desplazamientos automóviles forzados, ... Sin embargo, excepto en algunos locales asociativos, el desafío ecológico no es un objetivo principal de los locales compartidos.

El desafío está en diseñar estrategias de lugares compartidos a mayor escala, donde los objetivos medioambientales sean muy explícitos.

\subsection{Reto $n^{\circ}$ 11: Los modelos abiertos al servicio de la transición ecológica}

La complementariedad entre ecología y modelos abiertos mayoritariamente provenientes de la era digital resulta bastante clara: los modelos abiertos pueden ayudar a que proyectos con propósito ecológico pasen a mayor escala sin necesariamente abandonar sus principios y valores; Sin embargo, los modelos abiertos no son verdes por esencia: sin una intención explicita de impacto positivo sobre el medioambiente que además sea prioridad de la organización, los modelos abiertos no impactan positivamente sobre el medioambiente.

Para mejorar, el reto es doble: por una parte, se trata de sacar provecho de la experiencia en modelos abiertos para permitir el crecimiento de los proyectos ecológicos, que se repliquen, se 


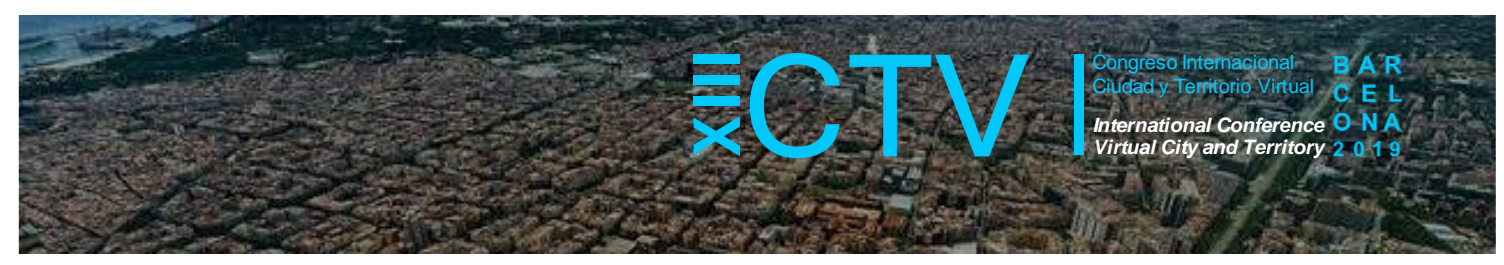

conecten, duren en el tiempo, ... Y, por otra parte, se trata de enriquecer los modelos abiertos con los procedimientos de evaluación y gestión de los impactos.

\subsection{Reto $n^{\circ} 12$ : Nuevas fuentes de inspiración para la economía circular}

La economía circular en cuya base predominan las herramientas digitales, es a menudo representada como una fuente de posible "ecologización" de la economía contemporánea. Sin embargo, las realizaciones concretas son comedidas y además focalizan en la disociación entre crecimiento y consumo de recursos perdiendo a menudo de vista el reto principal que consiste en reducir tanto los volúmenes producidos como los consumidos.

Sin integración de esta perspectiva, la aportación de la digitalización a la economía circular seguirá siendo limitada: optimizar los flujos de información y objetos seguirá siendo un campo fértil pero que no puede producir por si solo efectos medioambientales decisivos.

Para ser más fructífero ecológicamente, el ecosistema de la economía circular podría explorar tres vías: ver en la digitalización no sólo un instrumento de cálculo sino también un lugar para la experimentación continua de nuevos modelos económicos; volver circular a la propia economía digital; trabajar en formas de valorización de la economía circular que no se basen en un principio de acumulación de riquezas.

\subsection{Reto $n^{\circ}$ 13: Enlazar lo digital y el low tech}

Todo o casi enfrenta lo digital y los low tech: la huella ecológica de la digitalización es masiva y crece sin parar, el ritmo de renovación de sus utillajes es frenético. Resumidamente, lo más a menudo la digitalización promueve el crecimiento cuando los low tech abogan por la desobsolescencia, la reparabilidad y la economía de recursos.

Sin embargo, comparten un conjunto de valores: la colaboración entre compañeros; un patrimonio informativo común, compartido y cooperativo; una cultura del desvío y la experimentación.

En consecuencia, el reto no consiste en oponerles sino en buscar caminos donde su encuentro podría favorecer la consecución de retos ecológicos: idear otras formas de diseño, conectar recursos y comunidades para producir objetos de tipología distinta y más duraderos, construir idearios de una "frugalidad feliz" y concebir una digitalización enfocada a mejor en vez de a más, que se inspire de los principios que regentan los low tech.

\subsection{Reto $n^{\circ} 14:$ Movilizar lo digital al servicio de una democracia ecológica}

Los civic tech o grupos de compromiso cívico para una tecnología al servicio de los retos ecológicos existen en varios campos, ya sea para facilitar la confrontación de argumentos, mejorar el flujo de información o renovar las interacciones con los tomadores de decisiones.

Sin embargo, su contribución a la transición ecológica aún no es muy visible: su capacidad para producir empoderamiento es cuestionable, los modelos económicos a menudo se asocian con la venta de datos y su vínculo con los actores "tradicionales" de la democracia participativa sigue 


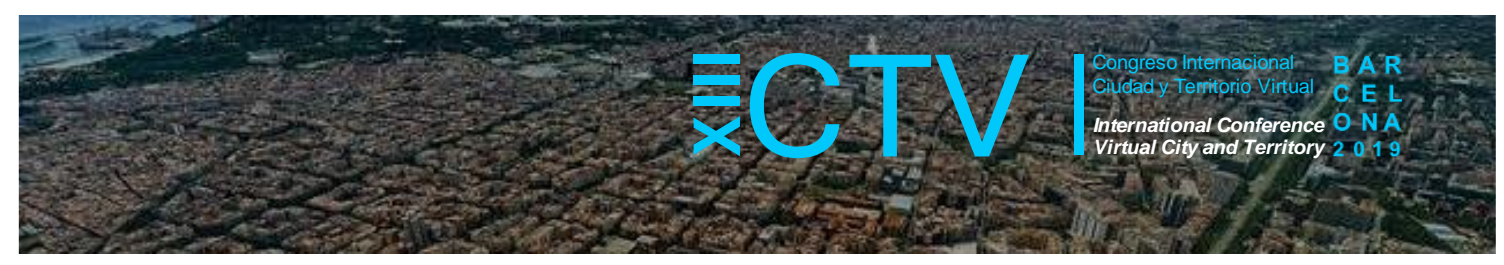

siendo débil. Los actores de los civic tech deben madurar, salir de una visión que a menudo es demasiado técnica y racional, con el riesgo de producir sistemas de toma de decisiones aún más incomprensibles. Con este objetivo, tendrán que trabajar más a fondo en una doble literatura, tanto digital como ecológica.

\subsection{Reto $n^{\circ} 15$ : Una segunda vida para ellos procomunes (o Commons en inglés) de la ecología}

Al facilitar la difusión de conocimientos, la digitalización ha reactivado la noción de procomún, también en asuntos medioambientales (cartografía del potencial de los paneles solares, seguimiento de la deforestación, licencias gratuitas y compartidas para proteger las semillas entre otros). Pero no basta con utilizar herramientas digitales con lógicas de compartir para convertirlos en procomunes y menos aún en un procomún virtuoso desde un punto de vista ambiental como lo ha demostrado la experiencia del consumo cooperativo.

Una agenda de lo digital al servicio del procomún ambiental debería incitar a una búsqueda de cultura compartida entre los actores de la digitalización, los de la ecología y los Commoners que suelen servirse en las dos culturas. Los actores digitales están acostumbrados a los nuevos modelos de producción y distribución del conocimiento y sus aplicaciones; los ecológicos tienen más práctica en los asuntos complicados de gobernanza, incluidos los de plazos alargados.

\section{Cinco emergencias a incluir en la agenda}

\subsection{Reto $n^{\circ} 16$ : Una cultura común de la transición digital y la ecología al servicio de} los Objetivos de Desarrollo Sostenible (ODS)

La contribución de la digitalización a los Objetivos de Desarrollo Sostenible se reduce demasiado a menudo a unas soluciones técnicas - la tecnología debe dar solución a los retos sociales y económicos - e inclusivas donde el objetivo es conectar a todos y cada uno a la infraestructura Internet para dar acceso al conocimiento de manera equitativa.

Pero por el carácter sistémico de los retos ecológicos, la aportación de la digitalización no puede reducirse a un conjunto de herramientas: si debe ayudar a resolver la crisis (en el sentido de metamorfosis) ecológica, tiene que sostener un cambio modal hacia nuevos modelos de desarrollo, nuevas formas de producción, de intercambio y de consumo.

Una agenda de la digitalización al servicio de los ODD debería comprometerse a un mejor uso de los datos, a desarrollar herramientas que sean un vector de comprensión de los retos ecológicos, que articulen la participación y ayuden a los innovadores a ubicarse y que, a la vez, tomen en cuenta las interacciones entre los retos económicos, medio ambientales y sociales identificados por los ODD.

\subsection{Reto $n^{\circ}$ 17: La contribución de la digitalización a la agricultura, a la agro-ecología y a la permacultura}

Drones, robots, estaciones agro-meteorológicas... la digitalización es, primero, un soporte para la racionalización de las decisiones y los esquemas predictivos, para permitir a los agricultores 


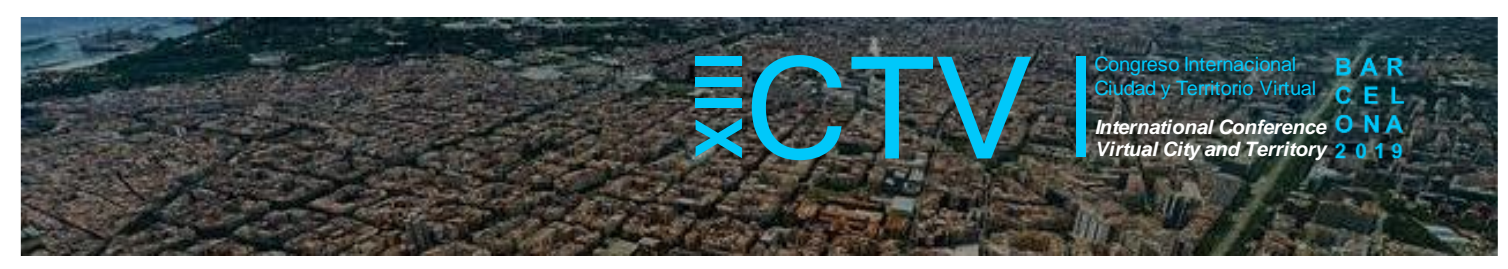

vigilar su producción et optimizar su rendimiento, lo que puede permitir la independencia tecnológica de estos agricultores. Pero la digitalización provee también herramientas para acompañar el resurgir de los circuitos cortos, de las redes locales de permacultura, de nuevas formas de alimentación, etcétera.

El reto está en dar más importancia a las iniciativas donde la digitalización sirve como herramienta para compartir conocimientos, para la conexión en red de iniciativas demasiado dispersas o para dar soporte a nuevos modelos, como por ejemplo los Open Models for Sustainability (modelos abiertos para la sostenibilidad).

\subsection{Reto $n^{\circ}$ 18: La digitalización al servicio de la vivienda sostenible}

Hoy el aporte de la digitalización a la vivienda sostenible está principalmente enfocado a la medida, la modelización y la eficiencia (BIM, Smart grids, ...).

Sin embargo, otras proposiciones con necesidades digitales ganarían al ser integradas en las estrategias de reducción de la huella medio ambiental de la vivienda y sus ocupantes: un uso mejor compartido de los datos, la consideración desde la concepción de los métodos de partición de los lugares, un mejor (re)conocimiento de las prácticas del DIY (Do It Yourself).

Una agenda de lo digital al servicio de la vivienda debería conectar los proyectos inteligentes con iniciativas más dispersas, basadas en la mutualización, el cooperativismo o la movilización.

\subsection{Reto $n^{\circ}$ 19: Hacia el Internet de la energía}

El Internet de la energía propone inspirarse en el modelo de internet para ingeniar sistemas descentralizados de distribución de energía, que se basen en buena parte en una gestión precisa de los datos.

A pesar del interés que suscita, este concepto es controvertido: se basa en tecnologías ya de por sí, muy consumidoras de recursos, no es suficiente para cubrir las necesidades de energía y además llevaría a un sobre-equipamiento, una sobre-producción o un sobre-consumo; por lo que sería beneficioso para los actores del sector más que para los consumidores.

Una agenda ecológica del internet de la energía podría basarse en buscar soluciones para reducir su propia huella ecológica y sobre todo a convertirlo en un proyecto colectivo de transformación de nuestra relación con la energía.

\subsection{Reto $n^{\circ} 20$ : Contra el colapso}

La digitalización está claramente designada como una de las causas del colapso en razón de varios factores: su huella ecológica y energética creciente, su marketing agresivo, y sobre todo porque es un símbolo de los métodos de producción y de consumo que no tienen en cuenta los límites del planeta. Sin embargo, es también una fuente de iniciativas (y también de imaginarios) que buscan integrar o empujar los límites planetarios, o que, hasta, se proyectan en un mundo post colapso: cultivar materiales, reproducir equilibrios en la biosfera, imaginar nuevas fuentes de alimentación, ... 


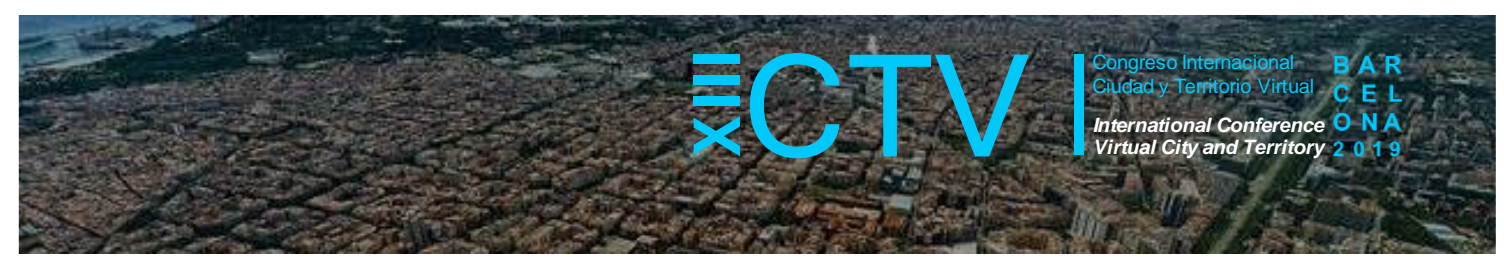

El reto está en construir una agenda híbrida, que integraría las iniciativas con alta densidad tecnológica (hoy, muchas de ellas son proyectos de investigación) a la vez que utilizaría la fuerza social de la digitalización para explorar nuevos imaginarios o apoyar el desarrollo de las low tech.

\section{Conclusiones}

¿Por qué interesarse por la digitalización cuando se busca integrar el desarrollo sostenible en la gobernanza y las políticas públicas? Sobre todo, porque la digitalización transforma nuestros modos de desplazamiento, de consumo, de producción, de aprendizaje, de participación al debate público, de elaboración de políticas, sobre todo a nivel de las ciudades.

La fructífera alianza entre la ecología y la tecnología digital invita a todos y cada uno a transformarse desde dentro. La Tech debe inventar una agenda de innovación que tenga en cuenta la finitud del mundo, y los ecologistas deben aprovechar el increíble poder de la transformación digital. No podemos pensar la sociedad del 2050 neutra en carbono y más justa sin pensar, a la vez, en como viviremos después de la transición digital.

En este sentido, los retos de la Agenda para un futuro digital y ecológico marcan los pasos para conseguir una digitalización innovadora a la vez que capacitadora, frugal, democrática, equitativa y factor de confianza. No cabe duda de que el potencial ecológico de esta digitalización es considerable y si consideramos que la problemática es mundial, interesa que el mensaje de la Agenda se propague fuera de los límites del hexágono.

Agradecimientos: A Jacques François Marchandise, secretario general de la FING por haber alentado la presente adaptación de su publicación, a los patrocinadores franceses de la FING y a los cerca de 500 participantes a las jornadas "Agenda para el Futuro Transitions2".

Contribuciones de los autores: Marike Charlier ha realizado la versión sintética en español de la agenda. Renaud Francou coordinó la publicación colectiva original en francés para la Fing y Transitions ${ }^{2}$ de L'agenda pour un futur numérique et écologique, 2019.

Conflicto de Intereses: Los autores declaran que no hay conflicto de intereses.

\section{Bibliografía}

Estalella A. (27 de mayo de 2013). El procomún no es un commons. Recuperado de http://www.prototyping.es/procomun/el-procomun-no-es-un-commons

Fing, Transitions. (2019). L'agenda pour un futur numérique et écologique. Recuperado de http://fing.org/IMG/pdf/Agenda-pour-un-futur-numerique-et-ecologique 2019 VF.pdf

Flok Society. (6 de marzo de 2014). ¿Qué es el procomún? What is a common? Recuperado de https://floksociety.org/2014/03/06/que-es-el-procomun/

Gutiérrez-Rubí, A. (7 de noviembre de 2018). Tecnología cívica para los desafíos urbanos. El País. Recuperado de https://elpais.com/elpais/2018/11/06/planeta futuro/1541496170 613226.html 